\title{
Hiding Charge in a Wormhole
}

\author{
Eduardo Guendelman ${ }^{*, 1}$, Alexander Kaganovich ${ }^{1}$ and Emil Nissimov², Svetlana Pacheva ${ }^{2}$ \\ ${ }^{1}$ Department of Physics, Ben-Gurion University of the Negev, P.O. Box 653, IL-84105, Beer-Sheva, Israel \\ ${ }^{2}$ Institute for Nuclear Research and Nuclear Energy, Bulgarian Academy of Sciences, Boul. Tsarigradsko Chausee 72, \\ BG-1784 Sofia, Bulgaria
}

\begin{abstract}
Existence of wormholes can lead to a host of new effects like Misner-Wheeler "charge without charge" effect, where without being generated by any source an electric flux arrives from one "universe" and flows into the other "universe". Here we show the existence of an intriguing opposite possibility. Namely, a charged object (a charged lightlike brane in our case) sitting at the wormhole "throat" expels all the flux it produces into just one of the "universes", which turns out to be of compactified ("tube-like") nature. An outside observer in the non-compact "universe" detects, therefore, a neutral object. This charge-hiding effect takes place in a gravity/gauge-field system self-consistently interacting with a charged lightlike brane as a matter source, where the gauge field subsystem is of a special non-linear form containing a square-root of the Maxwell term and which previously has been shown to produce a QCD-like confining gauge field dynamics in flat space-time.
\end{abstract}

PACS: 11.25.-w 04.70.-s 04.50.+h.

Keywords: Generalized Levi-Civita-Bertotti-Robinson spaces wormholes connecting non-compact with compactified "universes" wormholes via lightlike branes.

\section{INTRODUCTION}

Misner-Wheeler "charge without charge" effect [1] stands out as one of the most interesting physical phenomena produced by wormholes. Misner and Wheeler realized that wormholes connecting two asymptotically flat space times provide the possibility of existence of electromagnetically non-trivial solutions, where the lines of force of the electric field flow from one universe to the other without a source and giving the impression of being positively charged in one universe and negatively charged in the other universe. For a detailed account of the general theory of wormholes we refer to Visser's book [2] (see also [3, 4] and some more recent accounts [5-9].

In the present paper we find the opposite effect in wormhole physics, namely, that a genuinely charged matter source of gravity and electromagnetism may appear electrically neutral to an external observer. Here we show this phenomenon to take place in a gravity/gauge-field system self-consistently coupled to a charged lightlike brane as a matter source, where the gauge field subsystem is of a special non-linear form containing a square-root of the Maxwell term. The latter has been previously shown [10-15] to produce a QCD-like confining ("Cornell" [16-18]) potential in flat space-time. In the present case the lightlike brane, which connects as a wormhole "throat" a noncompact "universe" with a compactified "universe", is electrically charged, however all of its flux flows into the

*Address correspondence to this author at the Department of Physics, BenGurion University of the Negev, P.O. Box 653, IL-84105, Beer-Sheva, Israel; Tel: +972-8-647-2508; Fax: +972-8-647-2904;

E-mail: guendel@bgu.ac.il compactified ("tube-like") "universe" only. No Coulomb field is produced in the non-compact "universe", therefore, the wormhole hides the charge from an external observer in the latter "universe".

Let us recall that lightlike branes are singular null (lightlike) hypersurfaces in Riemannian space-time which provide dynamical description of various physically important phenomena in cosmology and astrophysics such as: (i) impulsive lightlike signals arising in cataclysmic astrophysical events (supernovae, neutron star collisions) [20]; (ii) dynamics of horizons in black hole physics -- the so called "membrane paradigm" [21]; (iii) the thin-wall approach to domain walls coupled to gravity [22-25]. The gravity/gauge-field system with a square-root of the Maxwell term was recently studied in [26] (see the brief review in Section 2 below) where the following interesting new features of the pertinent static spherically symmetric solutions have been found:

(i) appearance of a constant radial electric field (in addition to the Coulomb one) in charged black holes within Reissner-Nordström-de-Sitter-type and/or Reissner-Nordström- anti-de-Sitter-type space-times, in particular, in electrically neutral black holes with Schwarzschild-de-Sitter and/or Schwarzschild- antide-Sitter geometry;

(ii) novel mechanism of dynamical generation of cosmological constant through the nonlinear gauge field dynamics of the "square-root" Maxwell term;

(iii) appearance of confining-type effective potential in charged test particle dynamics in the above black hole backgrounds. 
In Section 3 of the present paper we extend the analysis in [26] by finding new solutions of Levi-Civita-BertottiRobinson type [27-29], i.e., with space-time geometry of the form $\mathcal{M}_{2} \times S^{2}$ with $\mathcal{M}_{2}$ being a two-dimensional anti-de Sitter, Rindler or de Sitter space depending on the relative strength of the electric field w.r.t. coupling of the square-root Maxwell term.

In our previous papers [30-40] we have provided an explicit reparametrization invariant world-volume Lagrangian formulation of lightlike $p$-branes (a brief review is given in Section 4) and we have used them to construct various types of wormhole, regular black hole and lightlike braneworld solutions in $D=4$ or higher-dimensional asymptotically flat or asymptotically anti-de Sitter bulk space-times. In particular, in refs. [38-40] we have shown that lightlike branes can trigger a series of spontaneous compactification-decompactification transitions of spacetime regions, e.g., from ordinary compactified ("tube-like") Levi-Civita-Bertotti-Robinson space to non-compact Reissner-Nordström or Reissner-Nordström-de-Sitter region or vice versa. Let us note that wormholes with "tube-like" structure (and regular black holes with "tube-like" core) have been previously obtained within different contexts in refs. [41-49].

The essential role of the above mentioned proper worldvolume Lagrangian formulation of lightlike branes manifests itself most clearly in the correct self-consistent construction $[34,37]$ of the simplest wormhole solution first proposed by Einstein and Rosen [50] -- the Einstein-Rosen "bridge" wormhole. Namely, in refs. $[34,37]$ it has been shown that the Einstein-Rosen "bridge" in its original formulation [50] naturally arises as the simplest particular case of static spherically symmetric wormhole solutions produced by lightlike branes as gravitational sources, where the two identical "universes" with Schwarzschild outer-region geometry are self-consistently glued together by a lightlike brane occupying their common horizon -- the wormhole "throat". An understanding of this picture within the framework of Kruskal-Szekeres manifold was subsequently provided in ref. [53], which involves Rindler's elliptic identification of the two antipodal future event horizons.

At this point let us strongly emphasize that the original notion of "Einstein-Rosen bridge" in ref. [50] is qualitatively different from the notion of "Einstein-Rosen bridge" defined in several popular textbooks (e.g., refs. [51, 52]) using the Kruskal-Szekeres manifold, where the "bridge" has dynamic space-time geometry. Namely, the two regions in KruskalSzekeres space-time corresponding to the two copies of outer Schwarzschild space-time region $(r>2 m)$ (the building blocks of the original static Einstein-Rosen "bridge") and labeled (I) and (III) in ref. [51] are generally disconnected and share only a two-sphere (the angular part) as a common border $(U=0, V=0$ in Kruskal-Szekeres coordinates), whereas in the original Einstein-Rosen "bridge" construction [50] the boundary between the two identical copies of the outer Schwarzschild space-time region $(r>2 m)$ is a threedimensional lightlike hypersurface $(r=2 m)$.
In Section 5 below we consider self-consistent coupling of gravity/gauge-field system with a square-root of the Maxwell term to a charged lightlike brane, which will serve as a matter source of gravity and (nonlinear) electromagnetism. In this Section we derive the main result of the present paper -- wormhole-like solutions joining a non-compact "universe" to a compactified ("tube-like") "universe" (of generalized Levi-Civita-Bertotti-Robinson type) via a wormhole "throat" realized by the charged lightlike brane, which completely hides its electric flux from an outside observer in the non-compact "universe". This new charge "confining" phenomena is entirely due to the presence of the "square-root" Maxwell term.

\section{LAGRANGIAN FORMULATION. SPHERICALLY SYMMETRIC SOLUTIONS}

We will consider the simplest coupling to gravity of the nonlinear gauge field system with a square-root of the Maxwell term known to produce QCD-like confinement in flat space-time [10-15]. The relevant action is given by (we use units with Newton constant $G_{N}=1$ ):

$$
\begin{array}{r}
S=\int d^{4} x \sqrt{-G}\left[\frac{R(G)}{16 \pi}+L\left(F^{2}\right)\right], \quad L\left(F^{2}\right)=-\frac{1}{4} F^{2}-\frac{f}{2} \sqrt{\varepsilon F^{2}}, \\
F^{2} \equiv F_{\kappa \lambda} F_{\mu v} G^{k \mu} G^{\lambda v}, \quad F_{\mu v}=\partial_{\mu} A_{v}-\partial_{v} A_{\mu} .
\end{array}
$$

Here $R(G)$ is the scalar curvature of the space-time metric $G_{\mu v}$ and $G \equiv \operatorname{det}\left\|G_{\mu v}\right\|$; the sign factor $\varepsilon= \pm 1$ in the square root term in (1) corresponds to "magnetic" or "electric" dominance; $f$ is a positive coupling constant. It is important to stress that we will not introduce any bare cosmological constant term.

Let us note that the Lagrangian $L\left(F^{2}\right)$ in (1) contains both the usual Maxwell term as well as a non-analytic function of $F^{2}$ and thus it is a non-standard form of nonlinear electrodynamics. In this way it is significantly different from the original purely "square root" Lagrangian $-\frac{f}{2} \sqrt{F^{2}}$ first proposed by Nielsen and Olesen [54] to describe string dynamics (see also refs. $[55,56])$. The natural appearance of the "square-root" Maxwell term in effective gauge field actions was further motivated by 't Hooft [19] who has proposed that such gauge field actions are adequate for describing confinement (see especially Eq.(5.10) in [19]). He has in particular described a consistent quantum approach in which "square-root" gauge-field terms play the role of "infrared counterterms". Also, it has been shown in first three refs. [10-15] that the square root of the Maxwell term naturally arises as a result of spontaneous breakdown of scale symmetry of the original scale-invariant Maxwell theory with $f$ appearing as an integration constant responsible for the latter spontaneous breakdown.

Let us also remark that one could start with the nonAbelian version of the gauge field action in (1). Since we will be interested in static spherically symmetric solutions, the non-Abelian gauge theory effectively reduces to an Abelian one as pointed out in the ref. [10]. 
The corresponding equations of motion read:

$R_{\mu \nu}-\frac{1}{2} G_{\mu v} R=8 \pi T_{\mu \nu}^{(F)}$,

where

$T_{\mu \nu}^{(F)}=L\left(F^{2}\right) G_{\mu \nu}-4 L^{\prime}\left(F^{2}\right) F_{\mu \kappa} F_{\nu \lambda} G^{\kappa \lambda}$,

and

$\partial_{v}\left(\sqrt{-G} L^{\prime}\left(F^{2}\right) F_{\kappa \lambda} G^{\mu \kappa} G^{v \lambda}\right)=0$,

where $L^{\prime}\left(F^{2}\right)$ denotes derivative w.r.t. $F^{2}$ of the function $L\left(F^{2}\right)$ in (1).

In our preceding paper [26] we have shown that the gravity-gauge-field system (1) possesses static spherically symmetric solutions with a radial electric field containing both Coulomb and constant vacuum pieces:

$F_{0 r}=\frac{\varepsilon_{F} f}{\sqrt{2}}+\frac{Q}{\sqrt{4 \pi} r^{2}} \quad, \quad \varepsilon_{F}=\operatorname{sign}(Q)$,

and the space-time metric:

$$
\begin{aligned}
& d s^{2}=-A(r) d t^{2}+\frac{d r^{2}}{A(r)}+r^{2}\left(d \theta^{2}+\sin ^{2} \theta d \phi^{2}\right), \\
& A(r)=1-\sqrt{8 \pi}|Q| f-\frac{2 m}{r}+\frac{Q^{2}}{r^{2}}-\frac{2 \pi f^{2}}{3} r^{2},
\end{aligned}
$$

is Reissner-Nordström-de-Sitter-type with dynamically generated effective cosmological constant $\Lambda_{\text {eff }}=2 \pi f^{2}$.

Appearance in (7) of a "leading" constant term different from 1 resembles the effect on gravity produced by a spherically symmetric "hedgehog" configuration of a nonlinear sigma-model scalar field with $S O(3)$ symmetry [57] (cf. also [58]).

\section{GENERALIZED LEVI-CIVITA-BERTOTTI- ROBINSON SPACE-TIMES}

Here we will look for static solutions of Levi-CivitaBertotti-Robinson type [27-29] of the system (2) -- (4), namely, with space-time geometry of the form $\mathcal{M}_{2} \times S^{2}$ where $\mathcal{M}_{2}$ is some two-dimensional manifold:

$$
\begin{aligned}
d s^{2}= & -A(\eta) d t^{2}+\frac{d \eta^{2}}{A(\eta)}+r_{0}^{2}\left(d \theta^{2}+\sin ^{2} \theta d \phi^{2}\right), \\
& -\infty<\eta<\infty, r_{0}=\text { const },
\end{aligned}
$$

and being:

either purely electric type, where the sign factor $\varepsilon=-1$ in the gauge field Lagrangian $L\left(F^{2}\right)(1)$ :

$F_{\mu \nu}=0$ for $\mu, v \neq 0, \eta \quad, \quad F_{0 \eta}=F_{0 \eta}(\eta)$;

- $\quad$ or purely magnetic type, where $\varepsilon=+1$ in (1):

$$
F_{\mu \nu}=0 \text { for } \mu, v \neq i, j \equiv \theta, \phi \quad, \quad \partial_{0} F_{i j}=\partial_{\phi} F_{i j}=0 .
$$

In the purely electric case (9) the gauge field equations of motion become:
$\partial_{\eta}\left(F_{0 \eta}-\frac{\varepsilon_{F} f}{\sqrt{2}}\right)=0 \quad, \quad \varepsilon_{F} \equiv \operatorname{sign}\left(F_{0 \eta}\right)$,

yielding a constant vacuum electric field:

$F_{0 \eta}=c_{F}=$ arbitrary const . read:

The (mixed) components of energy-momentum tensor (3)

$T_{0}^{(F) 0}=T_{\eta}^{(F) \eta}=-\frac{1}{2} F_{0 \eta}^{2} \quad, \quad T_{i j}^{(F)}=g_{i j}\left(\frac{1}{2} F_{0 \eta}^{2}-\frac{f}{\sqrt{2}}\left|F_{0 \eta}\right|\right)$

Taking into account (13), the Einstein eqs. (2) for (ij), where $R_{i j}=\frac{1}{r_{0}^{2}} g_{i j}$ because of the $S^{2}$ factor in (8), yield:

$\frac{1}{r_{0}^{2}}=4 \pi F_{0 \eta}^{2}$, i.e. $r_{0}=\frac{1}{2 \sqrt{\pi}\left|c_{F}\right|}$.

The (00) Einstein eq. (2) using the expression $R_{0}^{0}=-\frac{1}{2} \partial_{\eta}^{2} A$ (ref. [59]; see also [60]) becomes:

$\partial_{\eta}^{2} A=8 \pi\left(\left|c_{F} \| c_{F}\right|-\sqrt{2} f\right)$.

Therefore, we arrive at the following three distinct types of Levi-Civita-Bertotti-Robinson solutions for gravity coupled to the non-Maxwell gauge field system (1):

(i) $\quad \operatorname{Ad} S_{2} \times S^{2}$ with strong constant vacuum electric field $\left|F_{0 \eta}\right|=\left|c_{F}\right|>\sqrt{2} f$, where $A d S_{2}$ is two-dimensional anti-de Sitter space with:

$A(\eta)=4 \pi\left|c_{F}\right|\left(\left|c_{F}\right|-\sqrt{2} f\right) \eta^{2}$

in the metric (8), $\eta$ being the Poincare patch spacelike coordinate.

(ii)

$\operatorname{Rind}_{2} \times S^{2}$ with constant vacuum electric field $\left|F_{0 \eta}\right|=\left|c_{F}\right|=\sqrt{2} f$, where $\operatorname{Rind}_{2}$ is the flat twodimensional Rindler space with:

$A(\eta)=\eta$ for $0<\eta<\infty$ or $A(\eta)=-\eta$ for $-\infty<\eta<0(17)$

in the metric (8).

(iii) $d S_{2} \times S^{2}$ with weak constant vacuum electric field $\left|F_{0 \eta}\right|=\left|c_{F}\right|<\sqrt{2} f$, where $d S_{2}$ is two-dimensional de Sitter space with:

$A(\eta)=1-4 \pi\left|c_{F}\right|\left(\sqrt{2} f-\left|c_{F}\right|\right) \eta^{2}$

in the metric (8). For the special value $\left|c_{F}\right|=\frac{f}{\sqrt{2}}$ we recover the Nariai solution $[61,62]$ with $A(\eta)=1-2 \pi f^{2} \eta^{2}$ and equality (up to signs) among energy density, radial and transverse pressures: $\rho=-p_{r}=-p_{\perp}=\frac{f^{2}}{4}\left(T_{v}^{(F) \mu}=\operatorname{diag}\left(-\rho, p_{r}, p_{\perp}, p_{\perp}\right)\right)$. 
In all three cases above the size of the $S^{2}$ factor is given by (14). Solutions (17) and (18) are new ones and are specifically due to the presence of the non-Maxwell squareroot term (with $\varepsilon=-1$ ) in the gauge field Lagrangian (1).

In the purely magnetic case (10) the gauge field equations of motion (4):

$\partial_{v}\left[\sin \theta\left(1+\frac{f}{\sqrt{F^{2}}}\right) F^{\mu v}\right]=0$

yield magnetic monopole solution $F_{i j}=B r_{0}^{2} \sin \theta \varepsilon_{i j}$, where $B=$ const , irrespective of the presence of the non-Maxwell square-root term. However, the latter does contribute to the energy-momentum tensor:

$T_{0}^{(F) 0}=T_{\eta}^{(F) \eta}=-\frac{1}{2} B^{2}-f|B| \quad, \quad T_{i j}^{(F)}=\frac{1}{2} g_{i j} B^{2}$.

Taking into account (19), the Einstein eqs. (2) for (ij) yield (cf. (14)):

$\frac{1}{r_{0}^{2}}=4 \pi B^{2}+\sqrt{2} f|B|$,

whereas the mixed-component (00) Einstein eq. (2) gives $\partial_{\eta}^{2} A=8 \pi B^{2}$. Thus in the purely magnetic case we obtain only one solution -- $A d S_{2} \times S^{2}$ space-time with magnetic monopole where:

$A(\eta)=4 \pi B^{2} \eta^{2}$

in the metric (8) and the size of the $S^{2}$ factor is determined by (22).

\section{LAGRANGIAN FORMULATION OF LIGHTLIKE BRANE DYNAMICS}

In what follows we will consider gravity/gauge-field system self-consistently interacting with a lightlike $p$-brane (LL-brane for short) of codimension one $(D=(p+1)+1)$. In a series of previous papers [30-40] we have proposed manifestly reparametrization invariant world-volume Lagrangian formulation in several dynamically equivalent forms of $L L$-branes coupled to bulk gravity $G_{\mu \nu}$ and bulk gauge fields, in particular, electromagnetic field $A_{\mu}$. Here we will use our Polyakov-type formulation given by the world-volume action:

$$
\begin{aligned}
& S_{\mathrm{LL}} q=-\frac{1}{2} \int d^{p+1} \sigma T b_{0}^{\frac{p-1}{2}} \sqrt{-\gamma}\left[\gamma^{a b} \bar{g}_{a b}-b_{0}(p-1)\right], \\
& \bar{g}_{a b} \equiv \partial_{a} X^{\mu} G_{\mu v} \partial_{b} X^{\nu}-\frac{1}{T^{2}}\left(\partial_{a} u+q \mathcal{A}_{a}\right)\left(\partial_{b} u+q \mathcal{A}_{b}\right), \quad \mathcal{A}_{a} \equiv \partial_{a} X^{\mu} A_{\mu} .
\end{aligned}
$$

Here and below the following notations are used:

- $\quad \gamma_{a b}$ is the intrinsic Riemannian metric on the worldvolume with $\gamma=\operatorname{det}\left\|\gamma_{a b}\right\| ; g_{a b}$ is the induced metric on the world-volume:

$$
g_{a b} \equiv \partial_{a} X^{\mu} G_{\mu v}(X) \partial_{b} X^{v},
$$

which becomes singular on-shell (manifestation of the lightlike nature), $c f$. Eq. (29) below); $b_{0}$ is a positive constant measuring the world-volume "cosmological constant".

- $\quad X^{\mu}(\sigma)$ are the $p$-brane embedding coordinates in the bulk $D$-dimensional space-time with Riemannian metric $\left.G_{\mu v}(x)(\mu, v=0,1, \ldots, D-1) ;(\sigma) \equiv \sigma^{0} \equiv \tau, \sigma^{i}\right)$ with $\mathrm{i}=1, \ldots, p ; \partial_{a} \equiv \frac{\partial}{\partial \sigma^{a}}$.

$u$ is auxiliary world-volume scalar field defining the lightlike direction of the induced metric (see Eq.(29) below) and it is a non-propagating degree of freedom (ref.[40]).

- $\quad \mathrm{T}$ is dynamical (variable) brane tension (also a nonpropagating degree of freedom).

- Coupling parameter $q$ is the surface charge density of the LL-brane.

The corresponding equations of motion w.r.t. $X^{\mu}, u, \gamma_{\mathrm{ab}}$ and $T$ read accordingly (using short-hand notation (24)):

$\partial_{a}\left(T \sqrt{|\bar{g}|} \bar{g}^{a b} \partial_{b} X^{\mu}\right)++T \sqrt{|\bar{g}|} \bar{g}^{a b} \partial_{b} X^{\lambda} \partial_{a} X^{v} \Gamma_{\lambda v}^{\mu}$

$+\frac{q}{T} \sqrt{|\bar{g}|} \bar{g}^{a b} \partial_{a} X^{v}\left(\partial_{b} u+q \mathcal{A}_{b}\right) \mathcal{F}_{\lambda v} G^{\mu \lambda}=0$.

$\partial_{a}\left(\frac{1}{T} \sqrt{|\bar{g}|} \bar{g}^{a b}\left(\partial_{b} u+q \mathcal{A}_{b}\right)\right)=0, \quad \gamma_{a b}=\frac{1}{b_{0}} g_{a b}$.

$T^{2}+\varepsilon \bar{g}^{a b}\left(\partial_{a} u+q \mathcal{A}_{a}\right)\left(\partial_{b} u+q \mathcal{A}_{b}\right)=0$.

Here $\bar{g}=\operatorname{det}\left\|\bar{g}_{a b}\right\|$ and $\Gamma_{\lambda v}^{\mu}$ denotes the Christoffel connection for the bulk metric $G_{\mu \nu}$.

The on-shell singularity of the induced metric $g_{a b}$ (25), i.e., the lightlike property, directly follows Eq. (4) and the definition of $\bar{g}_{a b}(24)$ :

$$
g_{a b} \bar{g}^{b c}\left(\partial_{c} u+q \mathcal{A}_{c}\right)=0 \text {. }
$$

Explicit world-volume reparametrization invariance of the LL-brane action (23) allows to introduce the standard synchronous gauge-fixing conditions for the intrinsic worldvolume metric $\gamma_{00}=-1, \gamma_{0 i}=0(i=1, \ldots, p)$. which reduces Eqs. (27) -- (28) to the following relations:

$$
\begin{aligned}
& \frac{\left(\partial_{0} u+q \mathcal{A}_{0}\right)^{2}}{T^{2}}=b_{0}+g_{00}, \quad \partial_{i} u+q \mathcal{A}_{i}=\left(\partial_{0} u+q \mathcal{A}_{0}\right) g_{0 i}\left(b_{0}+g_{00}\right)^{-1}, \\
& g_{00}=g^{i j} g_{0 i} g_{0 j}, \partial_{0}\left(\sqrt{g^{(p)}}\right)+\partial_{i}\left(\sqrt{g^{(p)}} g^{i j} g_{0 j}\right)=0, g^{(p)} \equiv \operatorname{det}\left\|g_{i j}\right\|,
\end{aligned}
$$

(recall that $g_{00}, g_{0 i}, g_{i j}$ are the components of the induced metric (25); $g^{i j}$ is the inverse matrix of $g_{i j}$ ). Then, as shown in refs. [30-40], consistency of LL-brane dynamics in static "spherically-symmetric"-type backgrounds (in what follows we will use Eddington-Finkelstein coordinates, $\left.d t=d v-\frac{d \eta}{A(\eta)}\right):$ 
$d s^{2}=-A(\eta) d v^{2}+2 d v d \eta+C(\eta) h_{i j}(\theta) d \theta^{i} d \theta^{j} \quad, \quad F_{v \eta}=F_{v \eta}(\eta)$, rest $=0$

with the standard embedding ansatz:

$X^{0} \equiv v=\tau \quad, \quad X^{1} \equiv \eta=\eta(\tau) \quad, \quad X^{i} \equiv \theta^{i}=\sigma^{i} \quad(i=1, \ldots, p)$.

requires the corresponding background (31) to possess a horizon at some $\eta=\eta_{0}$, which is automatically occupied by the LL-brane, i.e.:

$\eta(\tau)=\eta_{0} \quad, \quad A\left(\eta_{0}\right)=0$.

This property is called "horizon straddling" according to the terminology of Ref. [23]. Similar "horizon straddling" has been found also for $L L$-branes moving in rotating axially symmetric (Kerr or Kerr-Newman) and rotating cylindrically symmetric black hole backgrounds [36, 37].

\section{SELF-CONSISTENT WORMHOLE-LIKE SOLUT- IONS WITH LL-BRANES}

Let us now consider a bulk gravity/gauge-field system in $D=4$ (1) self-consistently interacting with a $p=2 L L$ brane:

$S=\int d^{4} x \sqrt{-G}\left[\frac{R(G)}{16 \pi}-\frac{1}{4} F^{2}-\frac{f}{2} \sqrt{-F^{2}}\right]+S_{\mathrm{LL}}[q]$,

where $S_{\mathrm{LL}}[q]$ is the LL-brane world-volume action (23) (with $p=2$ ). It is now the LL-brane which will be the material and charge source for gravity and (nonlinear) electromagnetism.

The equations of motion resulting from (34) read:

$$
\begin{aligned}
& R_{\mu \nu}-\frac{1}{2} G_{\mu \nu} R=8 \pi\left[T_{\mu \nu}^{(F)}+T_{\mu \nu}^{\text {(brane })}\right], \\
& \partial_{\nu}\left(\sqrt{-G}\left(1-\frac{f}{\sqrt{-F^{2}}}\right) F_{\kappa \lambda} G^{\mu \kappa} G^{v \lambda}\right)+j_{(\text {brane })}^{\mu}=0,
\end{aligned}
$$

together with the LL-brane equations (26) -- (28). $T_{\mu v}^{(F)}$ is the same as in (3). The energy-momentum tensor and the charge current density of the $L L$-brane are straightforwardly derived from the underlying world-volume action (23):

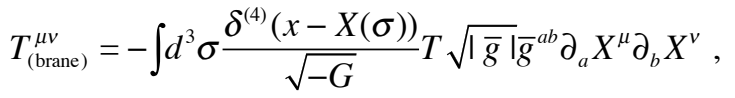

$$
\begin{aligned}
& j_{\text {(brane) }}^{\mu}=-q \int d^{3} \sigma \delta^{(4)}(x-X(\sigma)) \sqrt{|\bar{g}|} \bar{g}^{a b} \partial_{a} X^{\mu}\left(\partial_{b} u+q \mathcal{A}_{b}\right) T^{-1}
\end{aligned}
$$

Looking for solutions of static "spherically-symmetric"type (31) for the coupled gravity-gauge-field-LL-brane system (34) amounts to the following simple steps:

(i) Choose "vacuum" static "spherically-symmetric"type solutions (31) of (35) -- (36) (i.e., without the delta-function terms due to the LL-branes) in each region $-\infty<\eta<\eta_{0}$ and $\eta_{0}<\eta<\infty$ with a common horizon at $\eta=\eta_{0}$;

(ii) The LL-brane automatically locates itself on the horizon according to "horizon straddling" property (33); (iii) Match the discontinuities of the derivatives of the metric and the gauge field strength (31) across the horizon at $\eta=\eta_{0}$ using the explicit expressions for the LL-brane stress-energy tensor charge current density (37) -- (38).

Using (30) -- (32) we find for the LL-brane energymomentum tensor and charge current density:

$T_{\text {(brane) }}^{\mu v}=S^{\mu v} \delta\left(\eta-\eta_{0}\right), j_{\text {(brane) }}^{\mu}=\delta_{0}^{\mu} q \sqrt{\operatorname{det}\left\|G_{i j}\right\|} \delta\left(\eta-\eta_{0}\right)$,

where $G_{i j}=C(\eta) h_{i j}(\theta)(c f$. (31)). The non-zero components of the surface energy-momentum tensor $S_{\mu v}$ (with lower indices) and its trace are:

$S_{\eta \eta}=\frac{T}{b_{0}^{1 / 2}} \quad, \quad S_{i j}=-T b_{0}^{1 / 2} G_{i j} \quad, \quad S_{\lambda}^{\lambda}=-2 T b_{0}^{1 / 2}$.

Taking into account (39) -- (40) together with (31) -(33), the matching relations at the horizon $\eta=\eta_{0}$ become [38-40] (for a systematic introduction to the formalism of matching different bulk space-time geometries on codimension-one hypersurfaces ("thin shells") see the textbook [63]):

(A) Matching relations from Einstein eqs. (35):

$$
\left[\partial_{\eta} A\right]_{\eta_{0}}=-16 \pi T \sqrt{b_{0}} \quad, \quad\left[\partial_{\eta} \ln C\right]_{\eta_{0}}=-\frac{8 \pi}{\sqrt{b_{0}}} T
$$

with notation $Y_{\eta_{0}} \equiv Y \mathrm{I}_{\eta \rightarrow \eta_{0}+0}-Y \mathrm{I}_{\eta \rightarrow \eta_{0}-0}$ for any quantity $Y$.

(B) Matching relation from nonlinear gauge field eqs. (32):

$$
\left[F_{v \eta}\right]_{\eta 0}=q
$$

(C) $\quad X^{0}$-equation of motion of the LL-brane (the only non-trivial contribution of second-order LL-brane eqs. (26) in the case of embedding (32)):

$$
\begin{aligned}
& \frac{T}{2}\left(\left\langle\partial_{\eta} A\right\rangle_{\eta_{0}}+2 b_{0}\left\langle\partial_{\eta} \ln C\right\rangle_{\eta_{0}}\right)-\sqrt{b_{0}} q\left\langle F_{v \eta}\right\rangle_{\eta_{0}}=0 \\
& \text { with notation }\langle Y\rangle_{\eta_{0}} \equiv \frac{1}{2}\left(\left.Y\right|_{\eta \rightarrow \eta_{0}+0}+\left.Y\right|_{\eta \rightarrow \eta_{0}-0}\right) .
\end{aligned}
$$

We are looking for wormhole-type solutions to (34) with the charged LL-brane at the wormhole "throat" connecting a non-compact "universe" with Reissner-Nordstr"om-deSitter-type geometry (5)-(7) (where the cosmological constant is dynamically generated) to a compactified ("tubelike") "universe" of (generalized) Levi-Civita-BertottiRobinson type (8)-(9). These wormholes possess the novel property of hiding electric charge from external observer in the non-compact "universe", i.e., the whole electric flux produced by the charged LL-brane at the wormhole "throat" is pushed into the "tubelike" "universe".

The first wormhole-type solution of the above kind we find is given by:

(a) "left universe" of Levi-Civita-Bertotti-Robinson ("tube-like") type with geometry $\operatorname{Rind}_{2} \times S^{2}$ (17): 


$$
A(\eta)=-\eta \quad, \quad C(\eta)=r_{0}^{2} \quad, \quad\left|F_{v \eta}\right|=\sqrt{2} f \text { for } \eta<0 ;
$$

(b) non-compact "right universe" comprising the exterior region of Reissner-Nordström-de-Sitter-type black hole beyond the middle (Schwarzschild-type) horizon $r_{0}(c f .(5)--(7))$ :

$$
\begin{aligned}
& A(\eta)=1-\sqrt{8 \pi}|Q| f-\frac{2 m}{r_{0}+\eta}+\frac{Q^{2}}{\left(r_{0}+\eta\right)^{2}}-\frac{2 \pi f^{2}}{3}\left(r_{0}+\eta\right)^{2}, \\
& A(0)=0, \partial_{\eta} A(0)>0, \\
& C(\eta)=\left(r_{0}+\eta\right)^{2}, \quad F_{v \eta}=\frac{\varepsilon_{F} f}{\sqrt{2}}+\frac{Q}{\sqrt{4 \pi}\left(r_{0}+\eta\right)^{2}} \text { for } \eta>0 .(45)
\end{aligned}
$$

Substituting (44) -- (45) into the set of matching relations (41) -- (43) determines all parameters of the wormhole $\left(r_{0}, m, Q, b_{0}, q\right)$ in terms of the coupling constant $f$ in front of the square-root Maxwell term in (34):

$$
\begin{aligned}
& Q=0,|q|=\frac{f}{\sqrt{2}}, \operatorname{sign}(q)=-\operatorname{sign}\left(F_{v \eta}\right), \\
& r_{0}^{2}=\frac{1}{8 \pi f^{2}}, m=\frac{11}{48 \sqrt{2 \pi} f}, b_{0}=\frac{1}{8 \sqrt{2 \pi} f}+\frac{3}{16} .
\end{aligned}
$$

The second wormhole-type solution of the aforementioned kind reads:

(c) "left universe" of Levi-Civita-Bertotti-Robinson ("tube-like") type with geometry $A d S_{2} \times S^{2}$ (16):

$$
\begin{array}{r}
A(\eta)=4 \pi\left|c_{F}\right|\left(\left|c_{F}\right|-\sqrt{2} f\right) \eta^{2}, \quad C(\eta)=r_{0}^{2}, \\
\left|F_{v \eta}\right|=\left|c_{F}\right|>\sqrt{2} f \text { for } \quad \eta<0 ;
\end{array}
$$

(d) non-compact Reissner-Nordström-de-Sitter-type "right universe" of the same kind as (45).

Substituting again (48), (45) into the matching relations (41) -- (43) we find for the wormhole parameters:

$$
\begin{aligned}
& Q=0,\left|c_{F}\right|=|q|+\frac{f}{\sqrt{2}}, \operatorname{sign}(q)=-\operatorname{sign}\left(F_{v \eta}\right) \equiv-\operatorname{sign}\left(c_{F}\right), \\
& r_{0}^{2}=\frac{1}{4 \pi c_{F}^{2}}, m=\frac{1}{2 \sqrt{\pi} f}\left(1-\frac{f^{2}}{6 c_{F}^{2}}\right), b_{0}=\frac{|q|(|q|+\sqrt{2} f)}{4 c_{F}^{2}}
\end{aligned}
$$

The important observation here is that $Q=0$ in both wormhole solutions (a)-(b) (Eqs. (44) -- (45), (46) -- (47) and (c)-(d) (Eqs. (48), (45), (49) -- (50). Therefore, the "right universe" in both cases turns out to be the exterior region of the electrically neutral Schwarzschild-de-Sitter black hole beyond the Schwarzschild horizon which carries a vacuum constant radial electric field $\left|F_{v \eta}\right|=\frac{f}{\sqrt{2}}$. On the other hand, according to (45), (46) and (45), (49) the whole flux produced by the LL-brane charge $q \quad\left(\left|F_{v \eta}\right|=\frac{f}{\sqrt{2}}+|q|\right)$ flows only into the compactified "left universe" of Levi-
Civita-Bertotti-Robinson type $\left(\operatorname{Rind}_{2} \times S^{2} \quad\right.$ (17) or $A d S_{2} \times S^{2}(16)$ ).

The geometry of the above constructed charge-"hiding" wormhole solutions is illustrated in Fig. (1).

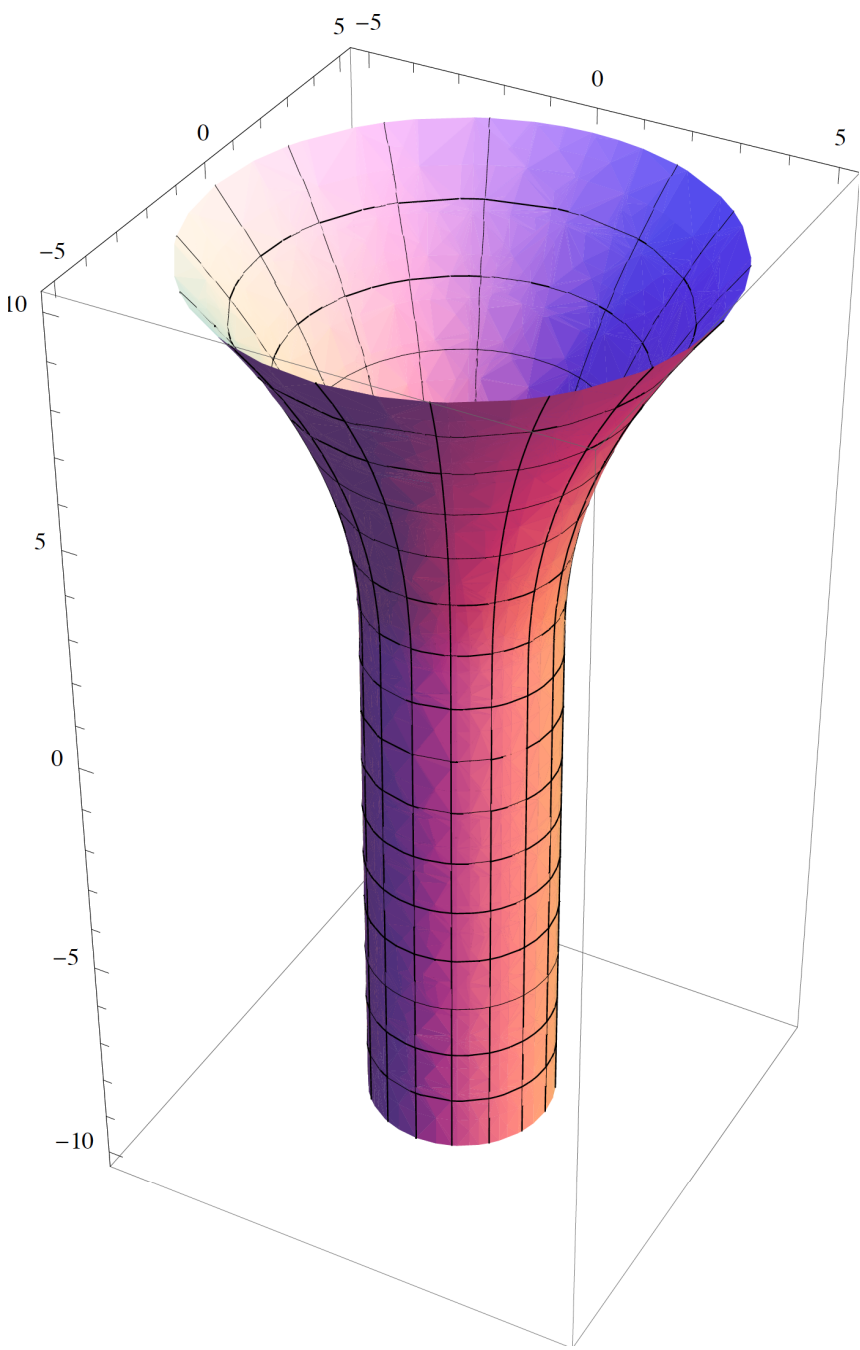

Fig. (1). Shape of $t=$ const and $\theta=\frac{\pi}{2}$ slice of charge-"hiding" wormhole geometry. The whole electric flux is expelled into the lower (infinitely long) cylindric tube.

\section{CONCLUSIONS}

We have seen that a charged wormhole "throat" realized by a charged lightlike brane, when joining a compactified space-time with a non-compact space-time region, expels all of the electric flux it produces into the compactified ("tubelike") region when the gauge field dynamics is driven by an additional "square-root" Maxwell term known to produce QCD-like confining potential in flat space-time. Indeed, this effect can be understood from the point of view of an observer in the non-compact "universe" as an alternative way of achieving charge confinement in a fashion similar to the MIT bag model [64], where the role of the inside bag region is being played by the compactified Levi-CivitaBertotti-Robinson space.

In an accompanying paper [65] we show that the above "charge-hiding" solution can be further generalized to a truly 
charge-confining wormhole solution when we couple the bulk gravity/nonlinear-gauge-field system (1) selfconsistently to two separate codimension-one charged lightlike branes with equal in magnitude but opposite charges. The latter system possesses a "two-throat" wormhole solution, where the "left-most" and the "rightmost" "universes" are two identical copies of the exterior region of the neutral Schwarzschild-de-Sitter black hole beyond the Schwarzschild horizon, whereas the "middle" "universe" is of generalized Levi-Civita-Bertotti-Robinson "tube-like" form with geometry $d S_{2} \times S^{2}$ (3). It comprises the finite-extent intermediate region of $d S_{2}$ between its two horizons. Both "throats" are occupied by the two oppositely charged lightlike branes and the whole electric flux produced by the latter is confined entirely within the middle finiteextent "tube-like" "universe" -- a property qualitatively resembling the quark confinement phenomenon in quantum chromodynamics.

\section{ACKNOWLEDGMENTS}

E.N. and S.P. are supported by Bulgarian NSF grant $D O$ 02-257. Also, all of us acknowledge support of our collaboration through the exchange agreement between the Ben-Gurion University and the Bulgarian Academy of Sciences. We are grateful to Stoycho Yazadjiev for constructive discussions. We also thank Doug Singleton for correspondence.

\section{CONFLICT OF INTEREST}

None declared.

\section{REFERENCES}

[1] Misner C, Wheeler JA. Classical physics as geometry gravitation, electromagnetism, unquantized charge, and mass as properties of curved empty space Ann of Phys 1957; 2: 525-603.

[2] Visser M. Lorentzian Wormholes. From Einstein to Hawking. Springer, Berlin; 1996.

[3] Hochberg D, Visser M. Geometric structure of the generic static traversable wormhole throat. Phys Rev 1997; D 56: 4745-55.

[4] Hochberg D, Visser M. Dynamic wormholes, antitrapped surfaces, and energy conditions. Phys Rev 1998; D 58: 044021-34.

[5] Lemos J, Lobo F, de Oliveira S. Morris-Thorne wormholes with a cosmological constant. Phys Rev 2003; D 68: 064004-18.

[6] Sushkov S. Wormholes supported by a phantom energy. Phys Rev 2005; D 71: 043520-24.

[7] Lobo F. Exotic solutions in general relativity: traversable wormholes and "warp drive" spacetimes. arxiv:0710.4474[gr-qc].

[8] Bronnikov B, Lemos J. Cylindrical wormholes. Phys Rev 2009; D 79: 104019-26.

[9] Bronnikov K, Skvortsova M, Starobinsky A. Notes on wormhole existence in scalar-tensor and $F(R)$ gravity. Grav Cosmol 2010; 16: 216-22.

[10] Gaete P, Guendelman E. Confinement from spontaneous breaking of scale symmetry. Phys Lett 2006; 640 B: 201-04.

[11] Gaete P, Guendelman E, Spalluci E. Static potential from spontaneous breaking of scale symmetry. Phys Lett 2007; 649 B: 217-24.

[12] Guendelman E. Scale symmetry breaking from the dynamics of maximal rank gauge field strengths. Int J Mod Phys 2004; A 19: 3255-64.

[13] Guendelman E. Strong interaction dynamics from spontaneous symmetry breaking of scale invariance. Mod Phys Lett 2007; A 22: 1209-16.

[14] Korover T, Guendelman E. Confinement effect as a result of spontaneous breaking of scale invariance. Int J Mod Phys 2009; A 24: 1443-56.
[15] Guendelman E. Bags and confinement governed by spontaneous ssymmetry breaking of scale invariance. Int J Mod Phys 2010; A 25: 4195-220.

[16] Eichten E, Gottfried K, Kinoshita T, Kogut J, Lane K, Yan T-M. The Spectrum of Charmonium. Phys Rev Lett 1975; 34: 369-72.

[17] Buchmüller W, Ed. Quarkonia, Current Physics Sources and Comments, vol.9, North Holland: 1992.

[18] Karliner M, Keren-Zur B, Lipkin H, Rosner J. The Quark Model and $b$ Baryons. Ann of Phys 2009; 324: 2-15.

[19] 't Hooft G. Perturbative confinement. Nucl Phys B (Proc. Suppl.) 2003; 121: 333-40.

[20] Barrabés C, Hogan P. Singular null-hypersurfaces in general relativity. World Scientific: Singapore; 2004.

[21] Thorne K, Price R, Macdonald D, editors. Black Holes: the membrane paradigm, Yale University Press: New Haven, CT; 1986.

[22] Israel W. Singular hypersurfaces and thin shells in general relativity. Nuovo Cim B 1966; 44: 1-14 (erratum. Nuovo Cim. B 1967; 48: 463).

[23] Barrabés $\mathrm{C}$, Israel $\mathrm{W}$. Thin shells in general relativity and cosmology: the lightlike limit. Phys Rev 1991; D 43: 1129-42.

[24] Dray T, 't Hooft G. The gravitational effect of colliding planar shells of matter. class. Quantum Grav 1986; 3: 825-40.

[25] Berezin V, Kuzmin A, Tkachev I. Dynamics of bubbles in general relativity. Phys Rev 1987; D 36; 2919-44.

[26] Guendelman E, Kaganovich A, Nissimov E, Pacheva S Asymptotically de Sitter and anti-de Sitter black holes with confining electric potential. Phys Lett 2011; 704 B: 230-3 (erratum. Phys Lett 2011; 705 B: 545).

[27] Levi-Civita T. The physical reality of some normal spaces of Bianchi. Rendiconti della Reale Accademia dei Lincei 1917; 26: 519-31.

[28] Bertotti B. Uniform electromagnetic field in the theory of general relativity. Phys Rev 1959; D 116: 1331-33.

[29] Robinson I. A Solution of the Maxwell-Einstein Equations. Bull Akad Pol 1959; 7: 351-2.

[30] Guendelman E, Kaganovich A, Nissimov E, Pacheva S. WeylConformally invariant light-like p-brane theories: new aspects in black hole physics and Kaluza-Klein dynamics. Phys Rev 2005; D 72: 0806011-23.

[31] Guendelman E, Kaganovich A, Nissimov E, Pacheva S. Weylinvariant lightlike branes and soldering of black hole space-times. Fortschr der Physik 2007; 55: 579-84.

[32] Guendelman E, Kaganovich A, Nissimov E, Pacheva S. 'Mass inflation' with lightlike branes. Centr Europ J Phys 2009; 7: 66876.

[33] Guendelman E, Kaganovich A, Nissimov E, Pacheva S. Lightlike branes as natural candidates for wormhole throats. Fortschr der Phys 2009; 57: 566-72.

[34] Guendelman E, Kaganovich A, Nissimov E, Pacheva S. EinsteinRosen "Bridge" needs lightlike brane source. Phys Lett 2009; 681 B: $457-62$.

[35] Guendelman E, Kaganovich A, Nissimov E, Pacheva S. Nonsingular black holes from gravity-matter-brane lagrangians. Int J Mod Phys 2010; A 25: 1571-96.

[36] Guendelman E, Kaganovich A, Nissimov E, Pacheva S. Variabletension lightlike brane as a gravitational source of traversable misner-wheeler-type wormholes. Phys Lett 2009; 673 B: 288-92.

[37] Guendelman E, Kaganovich A, Nissimov E, Pacheva S. Spherically symmetric and rotating wormholes produced by lightlike branes. Int J Mod Phys 2010; A 25: 1405-28.

[38] Guendelman E, Kaganovich A, Nissimov E, Pacheva S. Space-time compactification/decompactification transitions via lightlike branes. Gen Rel Grav 2011; 43: 1487-513.

[39] Guendelman E, Kaganovich A, Nissimov E, Pacheva S. Space-time compactification induced by lightlike branes. arxiv:0912.3712[hepth]).

[40] Guendelman E, Kaganovich A, Nissimov E, Pacheva S. Space-time compactification, non-singular black holes, wormholes and braneworlds via lightlike branes. in: Dragovic B, Rakic Z, ed. Sixth Meeting in Modern Mathematical Physics; Sept 2010; Belgrade Inst. Phys. Press, Belgrade, Serbia; 2011.

[41] Guendelman E. Wormholes and the construction of compactified phases. Gen Rel Grav 1991; 23: 1415-9.

[42] Dzhunushaliev V, Singleton D. Flux tube solutions in Kaluza-Klein theory. Class Quantum Grav 1999; 16: 973-8. 
[43] Dzhunushaliev V, Singleton D. Wormholes and flux tubes in 5-D Kaluza-Klein theory. Phys Rev 1999; D 59: 064018-23.

[44] Dzhunushaliev V, Kasper U, Singleton D. Gravitational flux tubes. Phys Lett 2000; 479 B: 249-56.

[45] Dzhunushaliev V. Delta string: a hybrid between wormhole and string. Gen Rel Grav 2003; 35: 1481-8.

[46] Matyjasek J, Zaslavsky O. Quantum back reaction of massive fields and selfconsistent semiclassical extreme black holes and acceleration horizons. Phys Rev 2001; D 64: 044005-15.

[47] Zaslavskii O. Classical model of elementary particle with BertottiRobinson core and extremal black holes. Phys Rev 2004; D 70: 104017-20.

[48] Zaslavskii O. N-spheres in general relativity: regular black holes without apparent horizons, static wormholes with event horizons and gravastars with a tube-like core. Phys Lett 2006; 634 B: 111-5.

[49] Zaslavskii O. Regular black holes with flux tube core. Phys Rev 2009; D 80: 064034-40.

[50] Einstein A, Rosen N. The particle problem in the general theory of relativity. Phys Rev 1935; 43: 73-7.

[51] Misner Ch, Thorne K, Wheeler JA. Gravitation. W.H. Freeman and Co: San Francisco, 1973.

[52] Carroll S. Spacetime and geometry. An introduction to general relativity. Addison Wesley: San Francisco, 2003.

[53] Poplawski N. Radial motion into an Einstein-Rosen Bridge. Phys Lett 2010; 687 B: 110-3.

[54] Nielsen H, Olesen P. Local field theory of the dual string. Nucl Phys 1973; B 57: 367-80.

[55] Aurilia A, Smailagic A, Spallucci E. Gauge theory of the string geodesic field. Phys Rev 1993; D 47: 2536-48.
[56] Amer N, Guendelman E. Flux tube solutions in square root gauge theory. Int J Mod Phys 2000; A 15: 4407-15.

[57] Guendelman E, Rabinowitz A. The Gravitational field of a hedgehog and the evolution of vacuum bubbles. Phys Rev 1991; D 44: 3152-8.

[58] Barriola M, Vilenkin A. Gravitational field of a global monopole. Phys Rev Lett 1989; 63: 341-3.

[59] Guendelman E, Rabinowitz A. Linearity, nonself interacting spherically symmetric gravitational fields, the sphereland equivalence principle and Hamiltonian bubbles. Gen Rel Grav 1996; $28: 117-28$.

[60] Jacobson T. When is $g_{t t} g_{r r}=-1$ ?. Class. Quantum Grav 2007; 24 : 5717-9.

[61] Nariai H. On a new cosmological solutions of Einstein's field equations of gravitation. Sci Rep Tohoku Univ. 1951; XXXV: 4657.

[62] Nariai H, Ueno Y. On some peculiar role of the cosmological constant in the general theory of relativity and in the Schroedinger theory of non-symmetric field. Progr Theor Phys 1960; 24: 114965.

[63] Poisson E. A relativist's tool kit. The Mathematics of black-hole mechanics. Cambridge Univ. Press: Cambridge UK, 2004.

[64] Chodos A, Jaffe RL, Johnson K, Thorn CB, Weisskopf V. A new extended model of Hadrons. Phys Rev 1974; D 9 3471-95.

[65] Guendelman E, Kaganovich A, Nissimov E, Pacheva S. Hiding and confining charges via "tube-like" wormholes. arxiv:1109.0453 [hep-th] (to appear in Int J Mod Phys A).

(c) Guendelman et al.; Licensee Bentham Open.

This is an open access article licensed under the terms of the Creative Commons Attribution Non-Commercial License (http://creativecommons.org/licenses/by$\mathrm{nc} / 3.0 /$ ) which permits unrestricted, non-commercial use, distribution and reproduction in any medium, provided the work is properly cited. 